\title{
Risk factors of regional lymph node (RLN) metastasis among patients with bone sarcoma and survival of patients with RLN- positive bone sarcoma
}

\author{
Yimin Dong ${ }^{1}$, Wei Wu ${ }^{1}$, Honglei Kang ${ }^{1}$, Wei Xiong ${ }^{1}$, Dawei Ye ${ }^{2}$, Zhong Fang ${ }^{1}$, Hanfeng Guan ${ }^{1}$, Hui Liao ${ }^{1}$, \\ Feng $\mathrm{Li}^{1}$ \\ ${ }^{1}$ Department of Orthopedics, Tongji Hospital, Tongji Medical College, Huazhong University of Science and Technology, Wuhan, China; ${ }^{2}$ Cancer \\ Center, Tongji Hospital, Tongji Medical college, Huazhong University of Science and Technology, Wuhan, China \\ Contributions: (I) Conception and design: F Li, H Liao; (II) Administrative support: None; (III) Provision of study materials or patients: None; (IV) \\ Collection and assembly of data: H Kang; (V) Data analysis and interpretation: Y Dong; W Wu; (VI) Manuscript writing: All authors; (VII) Final \\ approval of manuscript: All authors. \\ Correspondence to: Feng Li, MD; Hui Liao, MD. Department of orthopedics, Tongji Hospital, Tongji Medical College, Huazhong University of \\ Science and Technology, Wuhan, China. Email: lifengmd@hust.edu.cn; liaohui0001@yahoo.com.
}

Background: Regional lymph node metastasis (RLNM) has been reported to be a prognostic factor for poor survival outcomes of bone sarcoma. However, studies about risk factors for RLNM of bone sarcoma are extremely rare, and the outcome of such patients remains to be explored. We aimed to identify risk factors for RLNM of bone sarcoma and conduct survival analysis for patients with bone sarcoma with RLNM.

Methods: A total of 10,641 patients confirmed of malignant bone sarcomas from 1983 to 2014 were identified from the Surveillance, Epidemiology, and End Results (SEER) database, with 311 being regional lymph node positive. Logistic regression analysis was used to identify risk factors for RLNM, while the Cox proportional hazards model and the Fine and Gray's regression model were used for survival analysis.

Results: The proportion of RLNM was 6.0\% in Ewing sarcoma, 2.5\% in osteosarcoma and $1.1 \%$ in chondrosarcoma. Other bone tumors together had a RLNM rate of $4.2 \%$. Risk factors identified by the logistic regression analysis for RLNM were male patients, primary tumor site, tumor type and size. The multivariate Cox regression analysis suggested age, race, distant metastasis, tumor type and surgical treatment to be prognostic factors for the overall survival of patients with RLNM. Taking non-cancerspecific death as a competing risk, however, we found only age between 30-60 years [sub-distribution hazard ratio (SHR), 1.528, 95\% CI, 1.028-2.271; P=0.02], distant metastasis (SHR, 2.418, 95\% CI, 1.682-3.474; $\mathrm{P}<0.001)$ and surgery treatment $(\mathrm{SHR}, 0.493$, 95\% CI, 0.339-0.718; $\mathrm{P}<0.001)$ remained significant for the cancer-specific survival in the Fine and Gray's regression model.

Conclusions: Predictive factors for RLNM of bone sarcoma are sex, tumor site, type and size. In the presence of RKNM, only age, distant metastasis and surgery treatment are prognostic factors for the outcome of patients with bone sarcoma.

Keywords: Bone sarcoma; regional lymph node metastasis (RLNM); risk factors; survival; Surveillance, Epidemiology, and End Results (SEER)

Submitted Jun 13, 2020. Accepted for publication Sep 25, 2020.

doi: 10.21037/atm-20-4681

View this article at: http://dx.doi.org/10.21037/atm-20-4681 


\section{Introduction}

Malignant bone tumors are mainly sarcomas belonging to the mesenchymal tumor family, with osteosarcoma, Ewing sarcoma and chondrosarcoma being the three most common types (1). The survival of patients with bone sarcoma is largely dependent on the status of metastasis, which is generally considered to be achieved via hematogenous routes, with the lungs being the most common metastatic site (2). RLNM is relatively rare in bone sarcoma. Osteosarcoma, the most common type, has a RLNM rate of $1.4-2.3 \%$ in several studies $(3,4)$. For Ewing sarcoma and chondrosarcoma, the reported rate was $6.1 \%$ and $1.6 \%$ for primary tumor on the bone (5-7), while for primary tumor on extra-skeletal sites, the rate of RLNM is higher (31.1\% for Ewing sarcoma and 3.1\% for chondrosarcoma) $(8,9)$.

Despite the rare incidence, RLNM deserves great attention because it has been identified to be associated with significantly poor outcomes of patients with bone tumor $(5,9,10)$. For osteosarcoma, one animal study has suggested that RLNM is a prognostic factor for unfavorable outcomes (11). In human beings, the reported 5-year overall survival of patients with RLNM is $10.9 \%$, which is significantly lower than that $(54.3 \%)$ of patients without RLNM (10). For Ewing sarcoma, the second most common bone sarcoma in children, RLNM is more common when the sarcoma originates from extra-skeletal sties, and the 5 -year survival is decreased from $60.3 \%$ to $45.9 \%$ in the presence of RLNM (5). Chondrosarcoma mainly affects old people and is less malignant compared to the abovementioned two kinds of sarcoma. Over $80 \%$ of patients with chondrosarcoma can survive 10 years after treatment (12). However, patients may also suffer local recurrence and death within 2 years after treatment if regional lymph nodes are involved (7). These findings indicate that clinical attention should be paid to the status of RLNM.

Preoperative identification of patients at a high risk of RLNM can help surgeons take related measures to optimize the treatment for these patients. However, it is still difficult to recognize such high-risk patients. Factors associated with increased risks for RLNM of bone sarcoma are still not clear, and prognostic factors for the overall and cancerspecific survival also remain to be identified. Based on these considerations, we conducted this study to deeply explore risk factors for RLNM and performed survival analyses of bone sarcoma with RLNM, which may help clinical surgeons better deal with this disease.

We present the following article in accordance with the
STROBE reporting checklist (available at http://dx.doi. org/10.21037/atm-20-4681).

\section{Methods}

\section{Study population}

This retrospective study was conducted by using data from the Surveillance Epidemiology and End Results (SEER) database, which collects the clinical information of patients diagnosed with various cancers and covers approximately $30 \%$ of the total US population. The study was conducted in accordance with the Declaration of Helsinki (as revised in 2013), and individual consent for this retrospective analysis was waived as the patient data were extracted from the public SEER database. For our study, a total of 10,641 patients diagnosed with malignant bone sarcoma from 1983 to 2014 were finally enrolled from the database, with 311 having RLNM. Data of all patients were downloaded using the SEER*Stat software (version 8.3.5, National Cancer Institute, Bethesda, MD, USA). The diagnosis of each bone sarcoma was confirmed by positive histological examinations, while those diagnosed by positive exfoliative cytology, direct visualization or radiology were excluded. The final population to be investigated in this study was selected based on the following inclusion criteria: (I) malignant tumors diagnosed between 1983 and 2014, with the bone being the primary site; (II) confirmation of tumor by positive histology from living patients, not from biopsy or other dead cases; (III) known months of survival and complete follow-up information; (IV) known status of regional lymph node metastasis (RLNM); (V) known types of tumor and status of distant metastasis. A total of 10,641 patients meeting these criteria were finally included. Details of inclusion and exclusion processes are demonstrated in Figure 1.

\section{Variable definition}

Tumor type was determined according to the "ICD-O-3 Hist/behav" field in the SEER database, while RLNM information was obtained from the "EOD 4-nodes (19831987)", "EOD 10-nodes (1988-2003)" and "CS-lymph nodes (2004-2015)" fields for different periods of time. The treatment in this study was mainly local management such as radiotherapy and surgery. Surgery treatment was specific to cancer-directed surgeries, including tumor destruction, radical resection, limb salvage and amputation. Those 


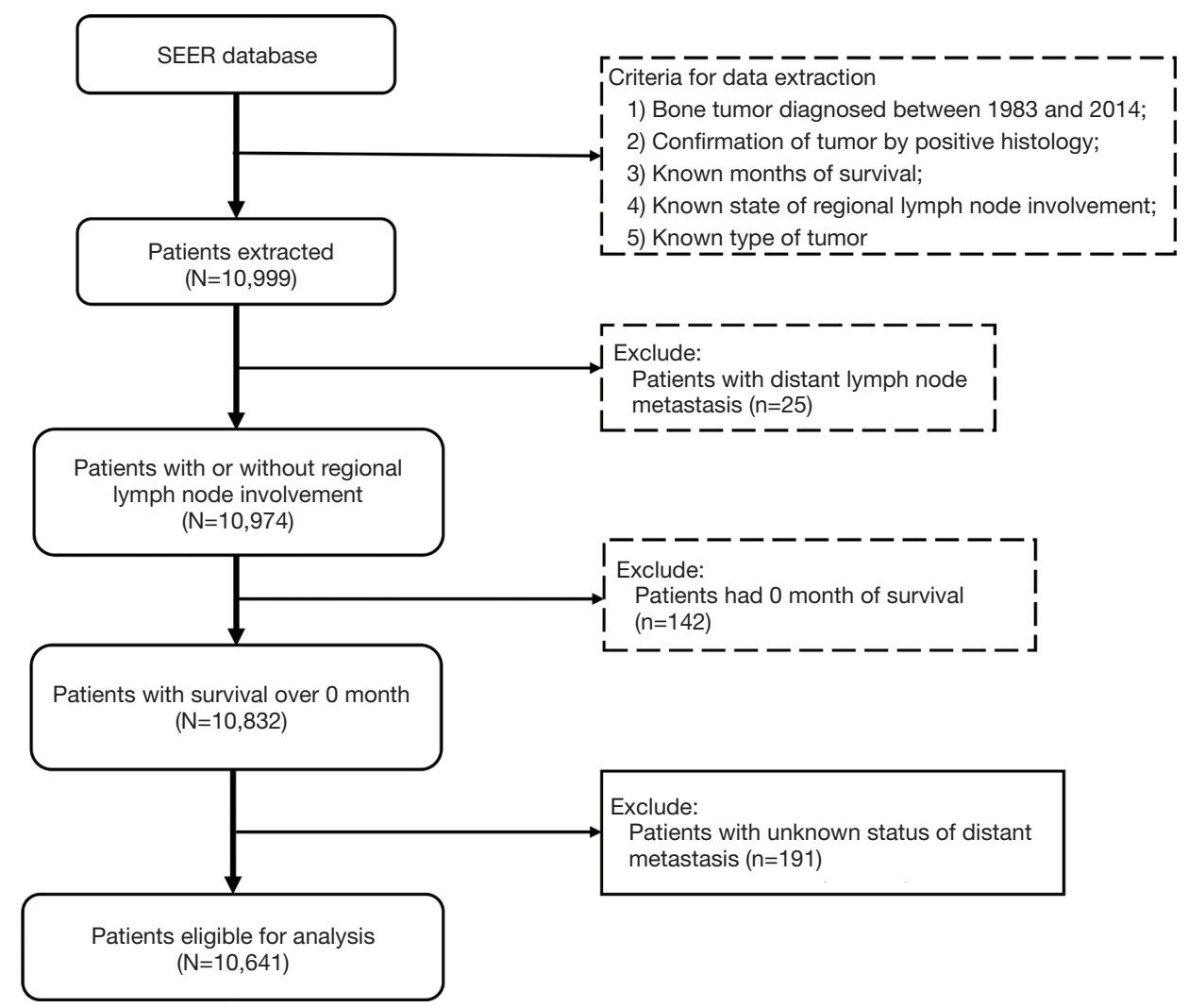

Figure 1 A flowchart demonstrating the inclusion criteria and exclusion process to the final study cohort.

without surgery treatment or undergoing non-cancerdirected procedures such as incisional, needle or aspiration biopsy were considered to be no-surgery-treated cases. Because chemotherapy was documented as "yes" and "no/ unknown" in SEER database, we did not know whether patients labeled as "unknown" received chemotherapy or not. In order to avoid possible bias in survival analysis, chemotherapy was excluded in the final multivariate analysis. Variables analyzed in our study included age, gender, race, tumor size, primary site, metastasis and treatment, most of which have been reported to be associated with the prognosis of bone sarcoma $(3,13)$. Age was grouped into $<30,30-60$, and $>60$ years. Tumor size was divided into four groups: $<6,6-12,>12 \mathrm{~cm}$ and unknown size. Metastatic information was obtained according to the SEER historic stage A [1973-2015] field. The primary outcome of interest was overall survival and cancer-specific survival. Overall survival was defined as survival span from first diagnosis to death of any cause, while cancer-specific survival was time from first diagnosis to death attributed to the primary bone sarcoma.

\section{Statistical analysis}

For baseline characteristics, categorical variates are presented as frequencies and percentages, whereas continuous variates are presented as median and interquartile range (IQR). Analysis of between-group difference in the baseline level was conducted by using the Wilcoxon rank-sum tests for continuous variates and the Chi-square test for categorical variates. In the survival analysis, age and tumor size were grouped as categorical variates. Univariate analysis was performed using the Kaplan-Meier curve and the log-rank test. Taking non-cancer-specific death as a competing risk to cancer-specific death, we plotted cumulative incidence function curves for each bone tumor and performed the Gray's test to compare the difference in the incidence rate of death (14). Multivariate analysis was performed using the Cox proportional hazards regression model and the Fine and Gray's regression model (14). Patients who were alive at last follow-up were considered as censored cases in the Cox analysis, while in the Fine and Gray's regression model, non-cancer-specific death was considered as the competing risk, and sub-distribution hazards ratio (SHR) was used to 


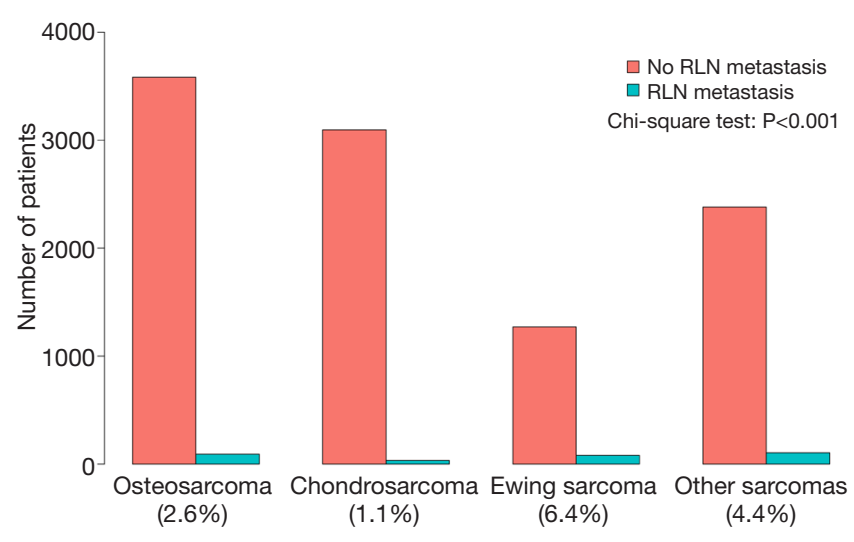

Figure 2 Rate of regional lymph node involvement for different bone sarcomas. Other sarcomas were calculated together due to low incidence. The difference of RLNM rate among different sarcomas was analyzed by chi-square test. RLNM, regional lymph node metastasis.

represent each variable's contribution to cancer-specific death. Statistical significance was defined as a $\mathrm{P}$ value less than 0.05 . All statistical analyses were performed in the $\mathrm{R}$ software (version 3.6.3). In the software, the "survival" package was used for the Kaplan-Meier curve and the Cox regression analysis, whereas the "timereg" and "cmprsk" packages were used for the competing risk analysis.

\section{Results}

\section{Demographics and clinical features of patients with or without RLNM}

The proportion of RLNM was $2.9 \%$ in the total cohort and was significantly different among different sarcomas (Figure 2, $\mathrm{P}<0.001$ ), with Ewing sarcoma having the highest $(6.0 \%)$ rate, followed by osteosarcoma (2.5\%), while chondrosarcoma had the lowest rate of RLNM (1.1\%). Other tumors together had a RLNM rate of $4.2 \%$. The total cohort was divided into a RLNM and a non-RLNM group. Baseline characteristics of patients in the two groups are presented in Table 1. Demographic characteristics such as age and race were similar between patients with and without RLNM, while male patients were more likely to have RLNM ( $65 \%$ vs. $56.2 \%, \mathrm{P}=0.003)$. RLNM tended to occur in bone sarcoma with a larger size [median (IQR), $9 \mathrm{~cm}$ $(5-14 \mathrm{~cm})$ vs. $7 \mathrm{~cm}(5-11 \mathrm{~cm}) ; \mathrm{P}<0.001]$, and the median survival in the RLNM group was significantly shorter [median (IQR), 17 months (7-44 months) vs. 54 months (21-114 months); $\mathrm{P}<0.001]$ compared to the non-RLNM group. The two groups also differed in the distribution of primary tumor sites, tumor types and treatments, with all p-values being less than 0.001. Distant metastasis was present in over half $(51.1 \%)$ of patients in the RLNM group, which was significantly higher than that in the nonRLNM group $(15 \%, \mathrm{P}<0.001)$.

\section{Risk factors for RLNM of bone sarcoma}

Univariate and multivariate logistic regression analyses were used to identify risk factors for RLNM. The factors with a $P$ value less than 0.1 in the univariate analysis were included in the final multivariate analysis to adjust for potential cofounding factors (Table 2). The result indicated that male patients [odds ratio (OR): 1.334, 95\% CI, 1.046-1.712; $\mathrm{P}=0.022]$, primary tumor site on head and face bones (OR: 1.778, 95\% CI, 1.287-2.461; $\mathrm{P}<0.001$ ), Ewing sarcoma (OR: $2.228,95 \% \mathrm{CI}, 1.556-3.185 ; \mathrm{P}<0.001)$, other sarcomas (OR: $1.744,95 \%$ CI, 1.234-2.469; $\mathrm{P}=0.002$ ) and tumor size $>12 \mathrm{~cm}$ (OR: 2.240, 95\% CI, 1.545-3.249; P<0.001) were associated with a higher risk of RLNM, while chondrosarcoma had a lower risk of RLNM compared to osteosarcoma (OR: 0.550, 95\% CI, 0.337-0.879; $\mathrm{P}=0.014$ ).

\section{Survival and outcome for bone sarcoma patients with RLNM}

Of the total cohort, the 311 patients with RLNM were subsequently included for survival analysis. The 5-year overall survival rate for osteosarcoma, chondrosarcoma and Ewing sarcoma with RLNM was $25.6 \%$ (95\% CI, $16.9-$ $38.7 \%$ ), $30.0 \%$ (95\% CI, $16.9-53.2 \%$ ) and $45.3 \%$ (95\% CI, $34.5-59.4 \%)$, respectively. Log-rank tests suggested that Ewing sarcoma was associated with a better overall survival compared to osteosarcoma $(\mathrm{P}<0.001)$ or chondrosarcoma $(\mathrm{P}=0.003)$, while no difference was observed between chondrosarcoma and osteosarcoma (OS: $\mathrm{P}=0.9$ ) (Figure 3). We also plotted cumulative incidence function curves considering non-cancer-specific death as the competing risk to the cancer-specific death. As shown in Figure 3, the cumulative incidence of cancer-specific death in Ewing sarcoma was lower than that in osteosarcoma (Gray's test, $\mathrm{P}=0.033$ ) but not in chondrosarcoma (Gray's test, $\mathrm{P}=0.181$ ). Unexpectedly, the incidence of the competing risk of Ewing sarcoma was also lower than that of osteosarcoma (Gray's test, $\mathrm{P}=0.004$ ) and chondrosarcoma (Gray's test, $\mathrm{P}=0.017$ ), suggesting that the difference of the overall survival between Ewing sarcoma and the other two sarcomas partially came 
Table 1 Characteristics of enrolled patients divided into non-RLNM and RLNM groups

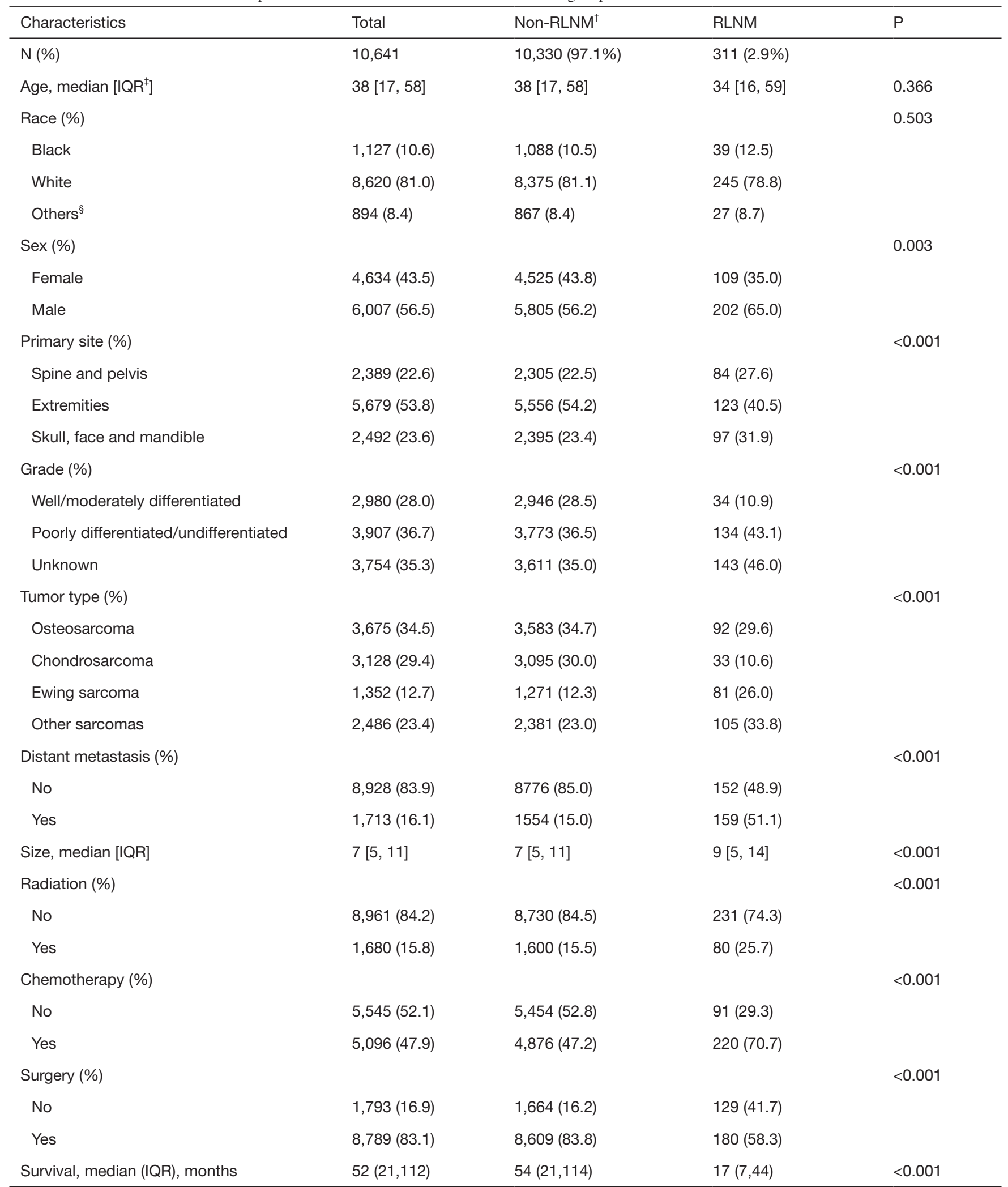

\footnotetext{
${ }^{\dagger}$, regional lymph node metastasis. ${ }^{\ddagger}$, interquartile range. ${ }^{\S}$, American Indian/Alaska Native, Asian/Pacific Islander. RLNM, regional lymph
} node metastasis 
Table 2 Logistic regression analysis for risk factors of regional lymph node metastasis

\begin{tabular}{|c|c|c|c|c|}
\hline \multirow{2}{*}{ Variables } & \multicolumn{2}{|c|}{ Univariate analysis } & \multicolumn{2}{|c|}{ Multivariate analysis } \\
\hline & OR $(95 \% \mathrm{Cl})^{\dagger}$ & $P$ & OR (95\% Cl) & $P$ \\
\hline \multicolumn{5}{|l|}{ Age, years } \\
\hline$<30$ & Reference & & Reference & \\
\hline $30-60$ & $0.759(0.581,0.988)$ & 0.042 & $1.089(0.794,1.486)$ & 0.594 \\
\hline \multicolumn{5}{|l|}{ Race } \\
\hline Black & Reference & & & \\
\hline White & $0.816(0.586,1.167)$ & 0.247 & & \\
\hline Others $^{\ddagger}$ & $0.869(0.523,1.424)$ & 0.58 & & \\
\hline \multicolumn{5}{|l|}{ Sex } \\
\hline Male & $1.445(1.143,1.835)$ & 0.002 & $1.334(1.046,1.712)$ & 0.022 \\
\hline \multicolumn{5}{|l|}{ Primary site } \\
\hline Spine and pelvis & Reference & & Reference & \\
\hline Extremities & $0.607(0.459,0.807)$ & 0.001 & $0.807(0.592,1.104)$ & 0.177 \\
\hline Skull, face and mandible & $1.111(0.826,1.499)$ & 0.487 & $1.778(1.287,2.461)$ & $<0.001$ \\
\hline \multicolumn{5}{|l|}{ Tumor type } \\
\hline Osteosarcoma & Reference & & Reference & \\
\hline Chondrosarcoma & $0.415(0.274,0.613)$ & $<0.001$ & $0.478(0.289,0.775)$ & 0.003 \\
\hline \multicolumn{5}{|l|}{ Grade } \\
\hline Well or moderately differentiated & Reference & & Reference & \\
\hline Poorly differentiated or undifferentiated & $3.077(2.132,4.566)$ & $<0.001$ & $1.466(0.948,2.317)$ & 0.093 \\
\hline Unknown & $3.431(2.384,5.081)$ & $<0.001$ & $1.132(0.727,1.805)$ & 0.591 \\
\hline \multicolumn{5}{|l|}{ Tumor size, $\mathrm{cm}$} \\
\hline$<6$ & Reference & & Reference & \\
\hline $6-12$ & $1.217(0.895,1.658)$ & 0.211 & $1.230(0.879,1.726)$ & 0.228 \\
\hline$>12$ & $2.395(1.736,3.303)$ & $<0.001$ & $2.240(1.545,3.249)$ & $<0.001$ \\
\hline Unknown & $1.227(0.880,1.707)$ & 0.225 & $1.070(0.748,1.528)$ & 0.709 \\
\hline
\end{tabular}

\footnotetext{
${ }^{\dagger}$, OR, odds ratio; $\mathrm{Cl}$, confidence interval. ${ }^{\ddagger}$, American Indian/Alaska Native, Asian/Pacific Islander.
} 

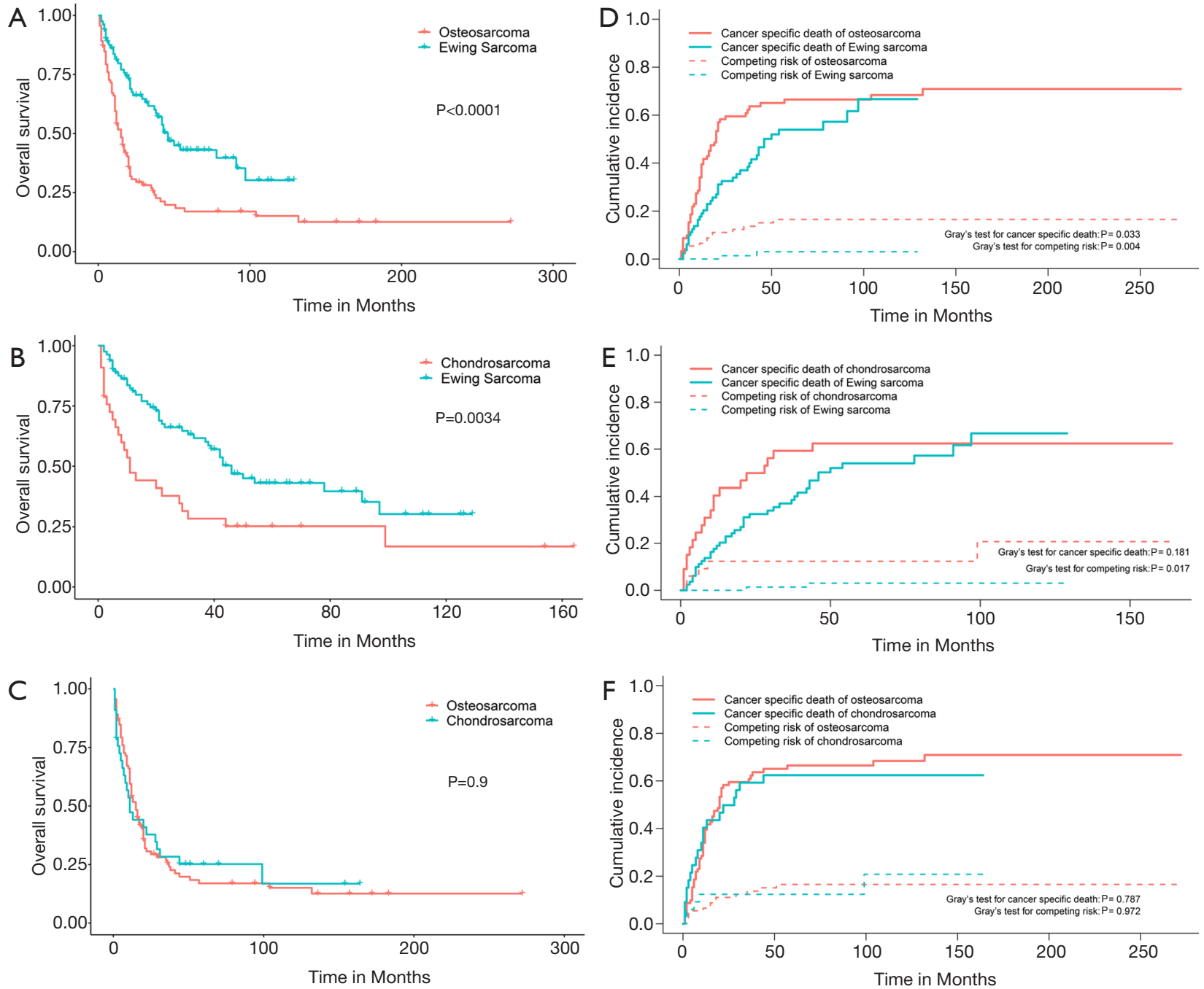

Figure 3 Kaplan-Meier curves and cumulative incidence function curves of bone sarcomas with regional lymph node involvement. Overall survival between osteosarcoma and Ewing sarcoma (A), chondrosarcoma and Ewing sarcoma (B), osteosarcoma and chondrosarcoma (C). The cumulative incidence of cancer-specific death and competing risks between osteosarcoma and Ewing sarcoma (D), chondrosarcoma and Ewing sarcoma (E), osteosarcoma and chondrosarcoma (F).

from non-cancer-specific death.

To adjust for potential confounding factors, multivariate Cox regressions for overall and cancer-specific survival were conducted by including all possible prognostic factors. The results suggested that age, race, distant metastasis and surgery were independent prognostic factors for both the overall and the cancer-specific survival (Table 3). Compared to Ewing sarcoma, chondrosarcoma was associated with a decreased overall survival (HR, 1.540, 95\% CI, 1.011-2.345; $\mathrm{P}=0.044)$ but not cancer-specific survival (HR, 1.377, 95\% CI, 0.877-2.163; $\mathrm{P}=0.165)$, and similar results were observed for other sarcomas. For osteosarcoma, there was no difference in the overall (HR, $1.627,95 \% \mathrm{CI}$, 0.914-2.896; $\mathrm{P}=0.098$ ) and cancer-specific survival (HR, 1.664, 95\% CI, 0.884-3.135; P=0.115) compared to Ewing sarcoma.

In the traditional Cox proportional hazards regression analysis for cancer-specific survival, patients dying of other causes are usually censured, leading to discrepancies because death of other causes precludes death of the primary tumor (15). Considering death not caused by specific bone sarcoma as the competing risk, we further conducted the Fine and Gray's regression analysis (Table 4). Significant factors associated with cancer-specific death after 
Table 3 Multivariate Cox analysis of overall and cancer-specific survival for 311 patients with RLNM

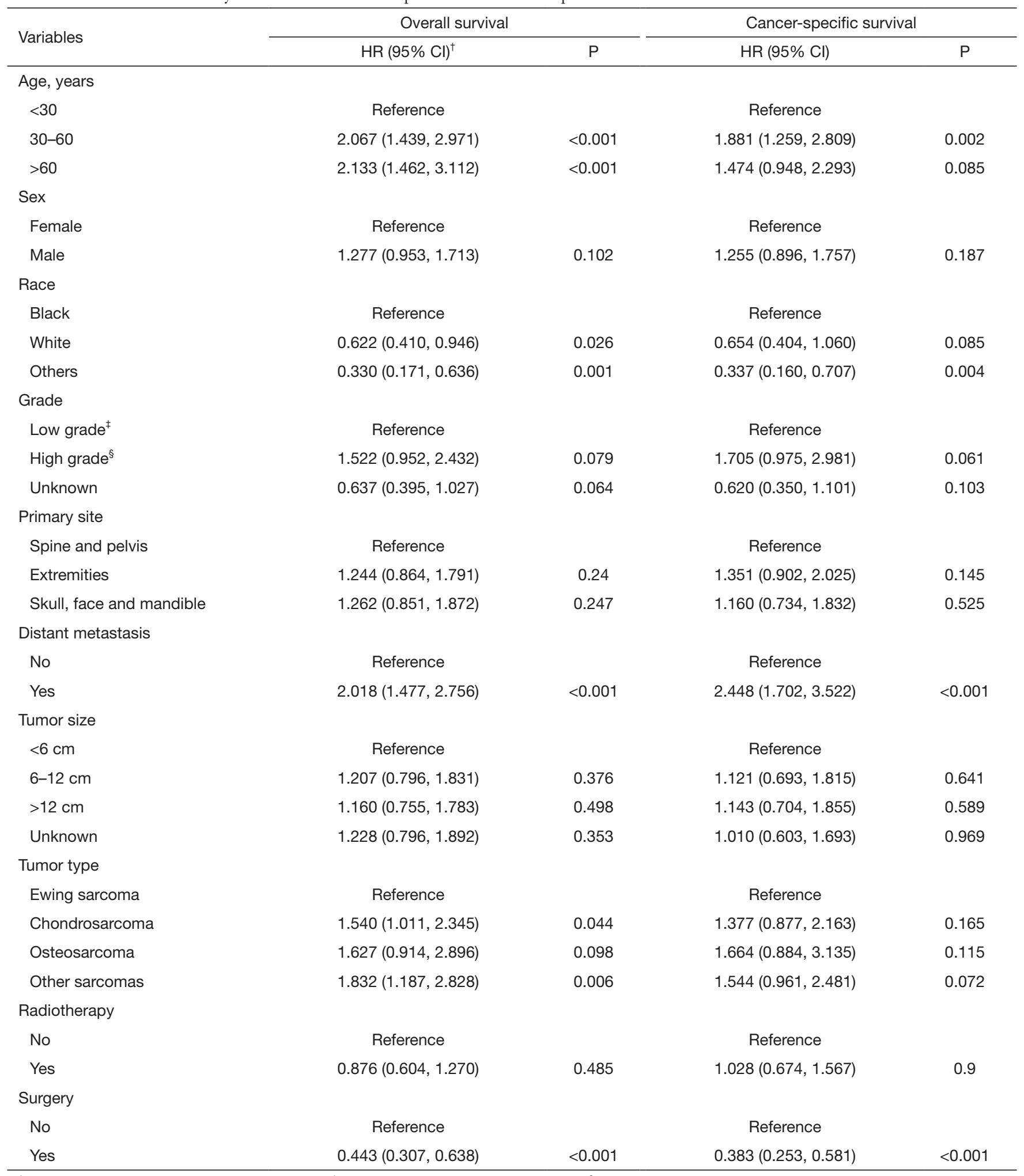

${ }^{\dagger}$, HR, hazard ratio; Cl, confidence interval. ${ }^{\ddagger}$, well or moderately differentiated. ${ }^{\S}$, poorly differentiated or undifferentiated. RLNM, regional lymph node metastasis. 
Table 4 Multiple competing risk regression analysis of patients with RLNM

\begin{tabular}{cc}
\hline Variables & $\begin{array}{c}\text { Sub-distribution hazard } \\
\text { ratio }\left(95 \% \mathrm{Cl}^{\dagger}\right)\end{array}$ \\
\hline
\end{tabular}

\begin{tabular}{|c|c|c|}
\hline \multicolumn{3}{|l|}{ Age, years } \\
\hline$<30$ & Reference & \\
\hline $30-60$ & $1.528(1.028,2.271)$ & 0.02 \\
\hline$>60$ & $0.958(0.610,1.505)$ & 0.91 \\
\hline \multicolumn{3}{|l|}{ Sex } \\
\hline Female & Reference & \\
\hline Male & $1.095(0.778,1.542)$ & 0.67 \\
\hline \multicolumn{3}{|l|}{ Race } \\
\hline Black & Reference & \\
\hline White & $0.953(0.544,1.668)$ & 0.87 \\
\hline Others & $0.511(0.232,1.125)$ & 0.08 \\
\hline \multicolumn{3}{|l|}{ Grade } \\
\hline Low grade f $^{\ddagger}$ & Reference & \\
\hline High grade ${ }^{\S}$ & $1.257(0.763,2.073)$ & 0.32 \\
\hline Unknown & $0.597(0.359,0.994)$ & 0.47 \\
\hline \multicolumn{3}{|l|}{ Primary site } \\
\hline Spine and pelvis & Reference & \\
\hline Extremities & $1.445(0.976,2.141)$ & 0.07 \\
\hline Skull, face and mandible & $1.086(0.670,1.762)$ & 0.96 \\
\hline \multicolumn{3}{|l|}{ Distant metastasis } \\
\hline No & Reference & \\
\hline Yes & $2.418(1.682,3.474)$ & $<0.001$ \\
\hline \multicolumn{3}{|l|}{ Tumor size, cm } \\
\hline$<6$ & Reference & \\
\hline $6-12$ & $1.225(0.732,2.048)$ & 0.44 \\
\hline$>12$ & $1.246(0.757,2.050)$ & 0.39 \\
\hline Unknown & & 0.96 \\
\hline \multicolumn{3}{|l|}{ Tumor type } \\
\hline Ewing sarcoma & Reference & \\
\hline Chondrosarcoma & $1.603(0.866,2.969)$ & 0.13 \\
\hline Osteosarcoma & $1.260(0.816,1.945)$ & 0.3 \\
\hline Other sarcomas & $1.382(0.875,2.184)$ & 0.17 \\
\hline \multicolumn{3}{|l|}{ Radiotherapy } \\
\hline No & Reference & \\
\hline Yes & $1.113(0.746,1.660)$ & 0.6 \\
\hline \multicolumn{3}{|l|}{ Surgery } \\
\hline No & Reference & \\
\hline Yes & $0.493(0.339,0.718)$ & $<0.001$ \\
\hline
\end{tabular}

${ }^{\dagger}$, Cl: confidence interval. ${ }^{\ddagger}$, well or moderately differentiated. ${ }^{\S}$, poorly differentiated or undifferentiated. RLNM, regional lymph node metastasis. controlling for competing risks were only age between 30-60 years (SHR, 1.528, 95\% CI, 1.028-2.271; P=0.02), distant metastasis (SHR, 2.418, 95\% CI, 1.682-3.474; $\mathrm{P}<0.001)$ and surgery treatment (SHR, $0.493,95 \% \mathrm{CI}$, $0.339-0.718 ; \mathrm{P}<0.001)$. There was no difference in the cancer-specific death among different bone sarcomas with RLNM (chondrosarcoma: SHR, 1.603, 95\% CI, 0.866-2.969, P=0.13; osteosarcoma: SHR, 1.260, 95\% CI, 0.816-1.945, $\mathrm{P}=0.13$; other sarcomas: SHR, $1.382,95 \% \mathrm{CI}$, $0.875-2.184, \mathrm{P}=0.17)$. Interestingly, tumor size was not an independent prognostic factor in all multivariate analyses.

To further confirm the credibility of the competing risk regression model, we conducted a series of sensitivity analyses, which included the following analyses: (I) analysis excluding patients without data of tumor size; (II) analysis excluding patients without tumor grade data; (III) analysis excluding patients with a survival less than 3 or 5 months to preclude the bias from surgery or other-treatment-related deaths; (IV) analysis including patients aged less than 30 years or older than 50 years to separately analyze young and old patients (Tables S1-S3). The results remained stable among these sensitivity analyses, except for that the survival of chondrosarcoma was poorer than Ewing sarcoma in patients younger than 30 years (SHR, 3.373, 95\% CI, 1.0510.77; $\mathrm{P}=0.04)$. Comprehensively, these results indicated that RLNM poses a similar cancer-specific outcome to patients with different bone sarcomas, and that patients with RLNM have a higher risk of death in the presence of distant metastasis. However, they can benefit from primarycancer-directed surgeries.

\section{Discussion}

Bone sarcomas are generally considered to metastasize via the hematogenous routes, mostly to the lungs and the bone $(2,16)$, while involvement of the lymph system is rare. Despite the rare incidence, the outcome of patients with RLNM is poorer than that in those without. Identifying patients at a high risk of RLNM is of great clinical significance, because it helps surgeons improve the treatment for such patients. However, to the best of our knowledge, there is no study available to explore risk factors for RLNM of bone sarcoma with relatively large size of sample. In addition, prognostic factors for patients with bone sarcoma with RLNM are also unclear. Considering this, we mined data from the SEER database and conducted this study, which is the first to provide a deep understanding of risk factors for RLNM and the survival outcomes of 
patients with bone sarcoma with RLNM.

The proportion of RLNM was significantly different among different bone sarcomas. Ewing sarcoma was found to have a higher rate of RLNM than osteosarcoma and other sarcomas, and chondrosarcoma was the least likely to have RLNM. This is consistent with previously reported rates of RLNM of different bone sarcomas (1.4-2.3\% for osteosarcoma, $1.6 \%$ for chondrosarcoma and $6.1 \%$ for Ewing sarcoma) from separate single centers $(3,4,6,7)$. The rate of RLNM for each sarcoma in our study is also similar to previous studies using data from the same database but including patients confirmed of bone sarcoma in different year spans $(5,9,10)$, which further adds to the credibility that the rate in our study represents the actual rate of RLNM for different bone sarcomas.

Using the multivariate logistic regression model, we found tumor type was a risk factor for RLNM. Ewing sarcoma and other bone tumors were more likely to metastasize to regional lymph nodes, while chondrosarcoma was associated with a lower risk of RLNM. This is in agreement with the different tendencies of distant metastasis for different bone sarcomas. The rate of distant metastasis at first diagnosis has been reported to be about 20\% for Ewing sarcoma, followed by $15 \%$ for osteosarcoma $(17,18)$. For chondrosarcoma, this rate decreases to less than 10\% (19), possibly due to the relatively low grade of malignancy and the less likelihood of metastasis of this sarcoma $(20,21)$. In the multivariate logistic analysis, distant metastasis was also suggestive of an increased risk for RLNM. These two types of metastasis might together reflect the divergent traits of metastasis of different bone sarcomas. Another important factor predictive of RLNM was larger tumor size $(>12 \mathrm{~cm})$, which has been reported to be a risk factor for RLNM of osteosarcoma (22). This reminds clinical surgeons that more attention should be paid to RLNM when resecting tumor with a large size.

Although Ewing sarcoma was more likely to have RLNM in this study, patients with such tumor had a better overall survival compared to other tumors with RLNM. However, such difference was eliminated in the cancerspecific survival regarding non-cancer-specific deaths as the competing risk, which suggests a comparable lethality of different tumors in the presence of RLNM. This further arouses concerns about the role of non-cancerspecific death in patient's survival. These deaths, including those attributed to cardiovascular events, diabetes or suicides, have been reported to be associated with the outcome of bone sarcoma or other cancers (23-25). Older age ( $>60$ years) was associated with a poor overall survival, but not cancer-specific survival for bone sarcoma patients with RLNM, which might also be attributed to the fact that old patients are at a higher risks of non-cancer-caused death (26).

Old-age-related non-cancer-specific death may also explain the different overall survival but not cancer-specific survival among different sarcomas, as osteosarcoma and chondrosarcoma have a relatively higher proportion of old patients compared to Ewing sarcoma, which affects mainly young patients (20). Actually, using the Gray's test, we found the cumulative incidence of non-cancer-specific death in Ewing sarcoma was significantly lower than that in osteosarcoma and chondrosarcoma. This difference is probably the cause of the different overall survival for patients with RLNM of different sarcomas. This reminds us that in addition to the treatment of tumor, preventing non-cancer-specific death in old patients is also a favorable strategy to improve the survival of patients with bone sarcoma.

The result of the multivariate competing risk regression model suggested that the cumulative incidence of cancerspecific death was not different in patients with different kinds of bone sarcoma. Chondrosarcoma is a less malignant tumor compared to other bone sarcomas. However, according to this study, RLNM confers equally poor outcomes to patients with chondrosarcoma compared to those with osteosarcoma and Ewing sarcoma. Tumor size, a risk factor for RLNM, was also not associated with the outcome of patients in the presence of RLNM. Small tumor is generally considered easy to resect and these patients are more likely to have a good outcome. However, our study shows that small tumor poses a comparable poor outcome to large tumor in the presence of RLNM. Cancer-directed surgery is associated with a decreased risk of cancer-specific death in the multivariate risk regression model, indicating that patients can benefit from surgery treatment. Thus, for patients at a high risk of RLNM, surgical treatment is recommended. In all, these findings remind doctors that the status of RLNM should be considered when resecting bone tumors, even for those with a small size or with a low-grade malignancy, to avoid potential poor outcomes associated with RLNM.

There were several limitations in this study. First, as lymph node examination is not a routine practice in bone tumor surgeries, the status of lymph node, the most important information for this study, was missing for a portion of patients. These patients were excluded from this 
study, causing difficulties in calculating the actual rate of RLNM. However, the rate of RLNM for different bone sarcomas analyzed in our study is similar to that of available single-center studies $(3,4)$. This convinced us that the rate would not change much when those excluded patients were included. Second, information of some key factors associated with the survival of bone sarcoma, such as tumor size and grade $(13,27)$, was not available for all patients included in the final analysis, which caused potential bias to the result. Considering this, we conducted related sensitivity analyses by excluding those patients missing information of tumor size or grade, and the results were in consistence with our main analysis. Third, although not effective for chondrosarcoma (28), chemotherapy is important for the treatment of osteosarcoma and Ewing sarcoma $(29,30)$. However, in the SEER database, chemotherapy information is unclearly documented, with those without chemotherapy and with unknown information of chemotherapy being labeled together as "no/unknown" in one group. We were unable to tell the patients truly without chemotherapy from other patients in the group, and chemotherapy was not included in our analysis. Consequently, we could not determine the role of chemotherapy on patients with bone sarcoma in the presence of RLNM. Further studies are needed to address the role of chemotherapy for such patients.

In conclusion, our study is the first to provide a deep understanding, including risk factors and survival analysis, for bone sarcoma with RLNM. As RLNM is associated with a significantly poor outcome for all bone sarcomas, more attention should be paid by clinical surgeons to the status of RLNM, especially for Ewing sarcoma, which has the highest rate of RLNM. Although chondrosarcoma is less malignant than other bone sarcomas, we should be cautious about the status of RLNM, which confers an equally poor survival compared to other bone sarcomas. It should be admitted that the rate of RLNM in our and other studies is based on non-routine clinical practices. Further studies are needed to explore the actual rate of RLNM for different sarcomas with intended regional lymph node examination. Considering that RLNM poses a significantly poorer outcome to patients with bone sarcoma, preoperative detection of RLNM and improvement of survival for these patients by surgical, radiological or chemotherapeutic strategies also deserve more research. Finally, non-cancerspecific death, which contributes to different overall-survival outcomes among different bone sarcomas with RLNM, may be focused on to improve the outcome of cancer patients.

\section{Acknowledgments}

We thank the clinical doctors and institutions who shared their data to the SEER database.

Funding: This work was supported by the National Natural Science Foundation of China (No. 81874024).

\section{Footnote}

Reporting Checklist: The authors have completed the STROBE reporting checklist. Available at http://dx.doi. org/10.21037/atm-20-4681

Peer Review File: Available at http://dx.doi.org/10.21037/ atm-20-4681

Conflicts of Interest: All authors have completed the ICMJE uniform disclosure form (available at http://dx.doi. org/10.21037/atm-20-4681). The authors have no conflicts of interest to declare.

Ethical Statement: The authors are accountable for all aspects of the work in ensuring that questions related to the accuracy or integrity of any part of the work are appropriately investigated and resolved. The study was conducted in accordance with the Declaration of Helsinki (as revised in 2013), and individual consent for this retrospective analysis was waived as the patient data were extracted from the public SEER database.

Open Access Statement: This is an Open Access article distributed in accordance with the Creative Commons Attribution-NonCommercial-NoDerivs 4.0 International License (CC BY-NC-ND 4.0), which permits the noncommercial replication and distribution of the article with the strict proviso that no changes or edits are made and the original work is properly cited (including links to both the formal publication through the relevant DOI and the license). See: https://creativecommons.org/licenses/by-nc-nd/4.0/.

\section{References}

1. Brown HK, Schiavone K, Gouin F, et al. Biology of Bone Sarcomas and New Therapeutic Developments. Calcif Tissue Int 2018;102:174-95.

2. Meazza C, Scanagatta P. Metastatic osteosarcoma: a challenging multidisciplinary treatment. Expert Rev Anticancer Ther 2016;16:543-56. 
3. Bielack SS, Kempf-Bielack B, Delling G, et al. Prognostic factors in high-grade osteosarcoma of the extremities or trunk: an analysis of 1,702 patients treated on neoadjuvant cooperative osteosarcoma study group protocols. J Clin Oncol 2002;20:776-90.

4. Tobias JD, Pratt CB, Parham DM, et al. The significance of calcified regional lymph nodes at the time of diagnosis of osteosarcoma. Orthopedics 1985;8:49-52.

5. Applebaum MA, Goldsby R, Neuhaus J, et al. Clinical features and outcomes in patients with Ewing sarcoma and regional lymph node involvement. Pediatr Blood Cancer 2012;59:617-20.

6. Mameghan H, Fisher RJ, O'Gorman-Hughes D, et al. Ewing's sarcoma: long-term follow-up in 49 patients treated from 1967 to 1989. Int J Radiat Oncol Biol Phys 1993;25:431-8.

7. Kurisunkal V, Gulia A, Puri A, et al. Lymph node metastasis in extremity chondrosarcomas: A series of four cases. South Asian J Cancer 2020;9:1-3.

8. Huh J, Kim KW, Park SJ, et al. Imaging Features of Primary Tumors and Metastatic Patterns of the Extraskeletal Ewing Sarcoma Family of Tumors in Adults: A 17-Year Experience at a Single Institution. Korean J Radiol 2015;16:783-90.

9. Wan L, Tu C, Li S, et al. Regional Lymph Node Involvement Is Associated With Poorer Survivorship in Patients With Chondrosarcoma: A SEER Analysis. Clin Orthop Relat Res 2019;477:2508-18.

10. Thampi S, Matthay KK, Goldsby R, et al. Adverse impact of regional lymph node involvement in osteosarcoma. Eur J Cancer 2013;49:3471-6.

11. Hillers KR, Dernell WS, Lafferty MH, et al. Incidence and prognostic importance of lymph node metastases in dogs with appendicular osteosarcoma: 228 cases (19862003). J Am Vet Med Assoc 2005;226:1364-7.

12. Angelini A, Guerra G, Mavrogenis AF, et al. Clinical outcome of central conventional chondrosarcoma. J Surg Oncol 2012;106:929-37.

13. Wang Z, Chen G, Chen X, et al. Predictors of the survival of patients with chondrosarcoma of bone and metastatic disease at diagnosis. J Cancer 2019;10:2457-63.

14. Fine JP, Gray RJ. A Proportional Hazards Model for the Subdistribution of a Competing Risk. J Am Stat Assoc 1999;94:496-509.

15. Yang L, Shen W, Sakamoto N. Population-based study evaluating and predicting the probability of death resulting from thyroid cancer and other causes among patients with thyroid cancer. J Clin Oncol 2013;31:468-74.

16. Ozaki T, Hillmann A, Lindner N, et al. Metastasis of chondrosarcoma. J Cancer Res Clin Oncol 1996;122:625-8.

17. ESMO Guidelines Working Group, Saeter G. Ewing's sarcoma of bone: ESMO clinical recommendations for diagnosis, treatment and follow-up. Ann Oncol 2007;18 Suppl 2:ii79-80.

18. Ritter J, Bielack SS. Osteosarcoma. Ann Oncol 2010;21:vii320-5.

19. Giuffrida AY, Burgueno JE, Koniaris LG, et al. Chondrosarcoma in the United States (1973 to 2003): an analysis of 2890 cases from the SEER database. J Bone Joint Surg Am 2009;91:1063-72.

20. Ferguson JL, Turner SP. Bone Cancer: Diagnosis and Treatment Principles. Am Fam Physician 2018;98:205-13.

21. Doyle LA. Sarcoma classification: an update based on the 2013 World Health Organization Classification of Tumors of Soft Tissue and Bone. Cancer 2014;120:1763-74.

22. Vasiliev NV. Lymph node metastasis of osteosarcomas. Arkh Patol 2016;78:58-63.

23. Luo J, Lin HC, He K, et al. Diabetes and prognosis in older persons with colorectal cancer. Br J Cancer 2014;110:1847-54.

24. Felix AS, Bower JK, Pfeiffer RM, et al. High cardiovascular disease mortality after endometrial cancer diagnosis: Results from the Surveillance, Epidemiology, and End Results (SEER) Database. Int J Cancer 2017;140:555-64.

25. Siracuse BL, Gorgy G, Ruskin J, et al. What is the Incidence of Suicide in Patients with Bone and Soft Tissue Cancer?: Suicide and Sarcoma. Clin Orthop Relat Res 2017;475:1439-45.

26. North BJ, Sinclair DA. The intersection between aging and cardiovascular disease. Circ Res 2012;110:1097-108.

27. Wang Z, Wu B, Zhou Y, et al. Predictors of the Survival of Primary and Secondary Older Osteosarcoma Patients. J Cancer 2019; 10:4614-22.

28. Gelderblom H, Hogendoorn PCW, Dijkstra SD, et al. The clinical approach towards chondrosarcoma. Oncologist 2008;13:320-9.

29. Yamamoto N, Tsuchiya H. Chemotherapy for osteosarcoma - where does it come from? What is it? Where is it going? Expert Opin Pharmacother 2013;14:2183-93.

30. Gaspar N, Hawkins DS, Dirksen U, et al. Ewing Sarcoma: Current Management and Future Approaches Through Collaboration. J Clin Oncol 2015;33:3036-46.

Cite this article as: Dong $\mathrm{Y}, \mathrm{Wu} \mathrm{W}$, Kang H, Xiong W, Ye D, Fang Z, Guan H, Liao H, Li F. Risk factors of regional lymph node (RLN) metastasis among patients with bone sarcoma and survival of patients with RLN-positive bone sarcoma. Ann Transl Med 2021;9(1):48. doi: 10.21037/atm-20-4681 


\section{Supplementary}

Table S1 Sensitivity analysis for survival of patients with RLNM by excluding those without tumor size or grade data

\begin{tabular}{|c|c|c|c|c|}
\hline \multirow{2}{*}{ Variables } & \multicolumn{2}{|c|}{ Excluding patients without tumor size data $(n=244)$} & \multicolumn{2}{|c|}{ Excluding patients without tumor grade data $(n=168)$} \\
\hline & $\operatorname{SHR}(95 \% \mathrm{Cl})^{\dagger}$ & $\mathrm{p}$ & SHR (95\% Cl) & $\mathrm{p}$ \\
\hline \multicolumn{5}{|l|}{ Age, years } \\
\hline$<30$ & Reference & & Reference & \\
\hline $30-60$ & $2.005(1.290,3.12)$ & 0.002 & $1.829(1.034,3.232)$ & 0.038 \\
\hline$>60$ & $1.240(0.740,2.08)$ & 0.41 & $1.089(0.630,1.884)$ & 0.76 \\
\hline \multicolumn{5}{|l|}{ Sex } \\
\hline Female & Reference & & Reference & \\
\hline Male & $1.167(0.778,1.75)$ & 0.46 & $1.258(0.806,1.963)$ & 0.31 \\
\hline \multicolumn{5}{|l|}{ Race } \\
\hline Black & Reference & & Reference & \\
\hline White & $0.989(0.519,1.89)$ & 0.97 & $1.075(0.547,2.115)$ & 0.83 \\
\hline Others & $0.445(0.186,1.07)$ & 0.069 & $0.383(0.150,0.976)$ & 0.044 \\
\hline \multicolumn{5}{|l|}{ Grade } \\
\hline Low grade & Reference & & Reference & \\
\hline High grade & $1.183(0.673,2.08)$ & 0.56 & $1.254(0.746,2.107)$ & 0.39 \\
\hline Unknown & $0.625(0.351,1.11)$ & 0.11 & & \\
\hline \multicolumn{5}{|l|}{ Primary site } \\
\hline Spine and pelvis & Reference & & Reference & \\
\hline Extremities & $1.570(0.979,2.52)$ & 0.061 & $1.683(0.969,2.922)$ & 0.064 \\
\hline Skull, face and, mandible & $1.076(0.596,1.94)$ & 0.81 & $0.931(0.492,1.761)$ & 0.83 \\
\hline \multicolumn{5}{|l|}{ Distant metastasis } \\
\hline No & Reference & & Reference & \\
\hline Yes & $2.482(1.639,3.76)$ & $<0.001$ & $3.182(1.971,5.136)$ & $<0.001$ \\
\hline \multicolumn{5}{|l|}{ Tumor, size } \\
\hline$<6 \mathrm{~cm}$ & Reference & & Reference & \\
\hline $6-12 \mathrm{~cm}$ & $1.187(0.679,2.07)$ & 0.55 & $1.089(0.551,2.149)$ & 0.81 \\
\hline$>12 \mathrm{~cm}$ & $1.285(0.752,2.19)$ & 0.36 & $1.310(0.700,2.453)$ & 0.4 \\
\hline Unknown & & & $1.466(0.751,2.861)$ & 0.26 \\
\hline \multicolumn{5}{|l|}{ Tumor type } \\
\hline Ewing sarcoma & Reference & & Reference & \\
\hline Chondrosarcoma & $1.050(0.499,2.21)$ & 0.9 & $1.388(0.647,2.974)$ & 0.4 \\
\hline Osteosarcoma & $1.146(0.678,1.94)$ & 0.61 & $1.035(0.616,1.740)$ & 0.9 \\
\hline Other sarcomas & $1.116(0.666,1.87)$ & 0.68 & $1.587(0.865,2.912)$ & 0.14 \\
\hline \multicolumn{5}{|l|}{ Radiotherapy } \\
\hline No & Reference & & Reference & \\
\hline Yes & $0.995(0.605,1.64)$ & 0.98 & $0.813(0.486,1.360)$ & 0.43 \\
\hline \multicolumn{5}{|l|}{ Surgery } \\
\hline No & Reference & & Reference & \\
\hline Yes & $0.522(0.340,0.80)$ & 0.003 & 0.509 (0.325 0.796) & 0.003 \\
\hline
\end{tabular}

${ }^{\dagger}$ SHR: Sub-distribution hazard ratio; $\mathrm{Cl}$ : confidence interval. 
Table S2 Sensitivity analysis for survival of patients with survival less than three or five months

\begin{tabular}{|c|c|c|c|c|}
\hline \multirow{2}{*}{ Variables } & \multicolumn{2}{|c|}{ Excluding survival less than three months $(n=269)$} & \multicolumn{2}{|c|}{ Excluding survival less than five months $(n=244$} \\
\hline & $\operatorname{SHR}(95 \% \mathrm{Cl})^{\dagger}$ & $\mathrm{p}$ & SHR (95\% Cl) & $\mathrm{p}$ \\
\hline \multicolumn{5}{|l|}{ Age, years } \\
\hline$<30$ & Reference & & Reference & \\
\hline $30-60$ & $1.316(0.8272 .094)$ & 0.25 & $1.263(0.7562 .110)$ & 0.37 \\
\hline$>60$ & $0.981(0.5971 .611)$ & 0.94 & $0.880(0.5141 .508)$ & 0.64 \\
\hline \multicolumn{5}{|l|}{ Sex } \\
\hline Female & Reference & & Reference & \\
\hline Male & $1.084(0.7371 .595)$ & 0.68 & $1.149(0.7511 .758)$ & 0.52 \\
\hline \multicolumn{5}{|l|}{ Race } \\
\hline Black & Reference & & Reference & \\
\hline White & $0.834(0.4741 .467)$ & 0.53 & $0.730(0.3711 .436)$ & 0.3 \\
\hline Others & $0.323(0.1370 .760)$ & 0.009 & $0.276(0.1050 .723)$ & 0.004 \\
\hline \multicolumn{5}{|l|}{ Grade } \\
\hline Low grade & Reference & & Reference & \\
\hline High grade & $1.384(0.7892 .428)$ & 0.26 & 1.225 (0.636 2.359) & 0.39 \\
\hline Unknown & $0.494(0.2720 .898)$ & 0.023 & $0.483(0.2490 .937)$ & 0.017 \\
\hline \multicolumn{5}{|l|}{ Primary site } \\
\hline Spine and pelvis & Reference & & Reference & \\
\hline Extremities & $1.218(0.8071 .837)$ & 0.35 & $1.327(0.8202 .147)$ & 0.25 \\
\hline Skull, face and mandible & $0.860(0.5201 .422)$ & 0.56 & $1.062(0.6191 .823)$ & 0.83 \\
\hline \multicolumn{5}{|l|}{ Distant metastasis } \\
\hline No & Reference & & Reference & \\
\hline Yes & 0.472 (0.307 0.728) & $<0.001$ & 2.474 (1.566 3.910) & $<0.001$ \\
\hline \multicolumn{5}{|l|}{ Tumor size } \\
\hline$<6 \mathrm{~cm}$ & Reference & & Reference & \\
\hline $6-12 \mathrm{~cm}$ & $1.120(0.6301 .988)$ & 0.7 & 1.095 (0.585 2.050) & 0.99 \\
\hline$>12 \mathrm{~cm}$ & $1.262(0.7432 .143)$ & 0.39 & $1.266(0.7082 .261)$ & 0.52 \\
\hline Unknown & $0.837(0.4631 .515)$ & 0.56 & $0.632(0.3201 .247)$ & 0.22 \\
\hline \multicolumn{5}{|l|}{ Tumor type } \\
\hline Ewing sarcoma & Reference & & Reference & \\
\hline Chondrosarcoma & 1.509 (0.786 2.897) & 0.22 & $2.000(0.9584 .177)$ & 0.06 \\
\hline Osteosarcoma & $1.355(0.8462 .171)$ & 0.21 & $1.601(0.9242 .774)$ & 0.09 \\
\hline Other sarcomas & 1.135 (0.682 1.889) & 0.63 & $1.204(0.6852 .118)$ & 0.52 \\
\hline \multicolumn{5}{|l|}{ Radiotherapy } \\
\hline No & Reference & & Reference & \\
\hline Yes & 1.176 (0.736 1.878) & 0.5 & 1.208 (0.729 2.001) & 0.46 \\
\hline \multicolumn{5}{|l|}{ Surgery } \\
\hline No & Reference & & Reference & \\
\hline Yes & $0.472(0.3070 .728)$ & $<0.001$ & $0.385(0.2300 .643)$ & $<0.001$ \\
\hline
\end{tabular}

${ }^{\dagger}$ SHR: Sub-distribution hazard ratio; Cl: confidence interval. 
Table S3 Sensitivity analysis for survival of patients aged less than 30 or over 50

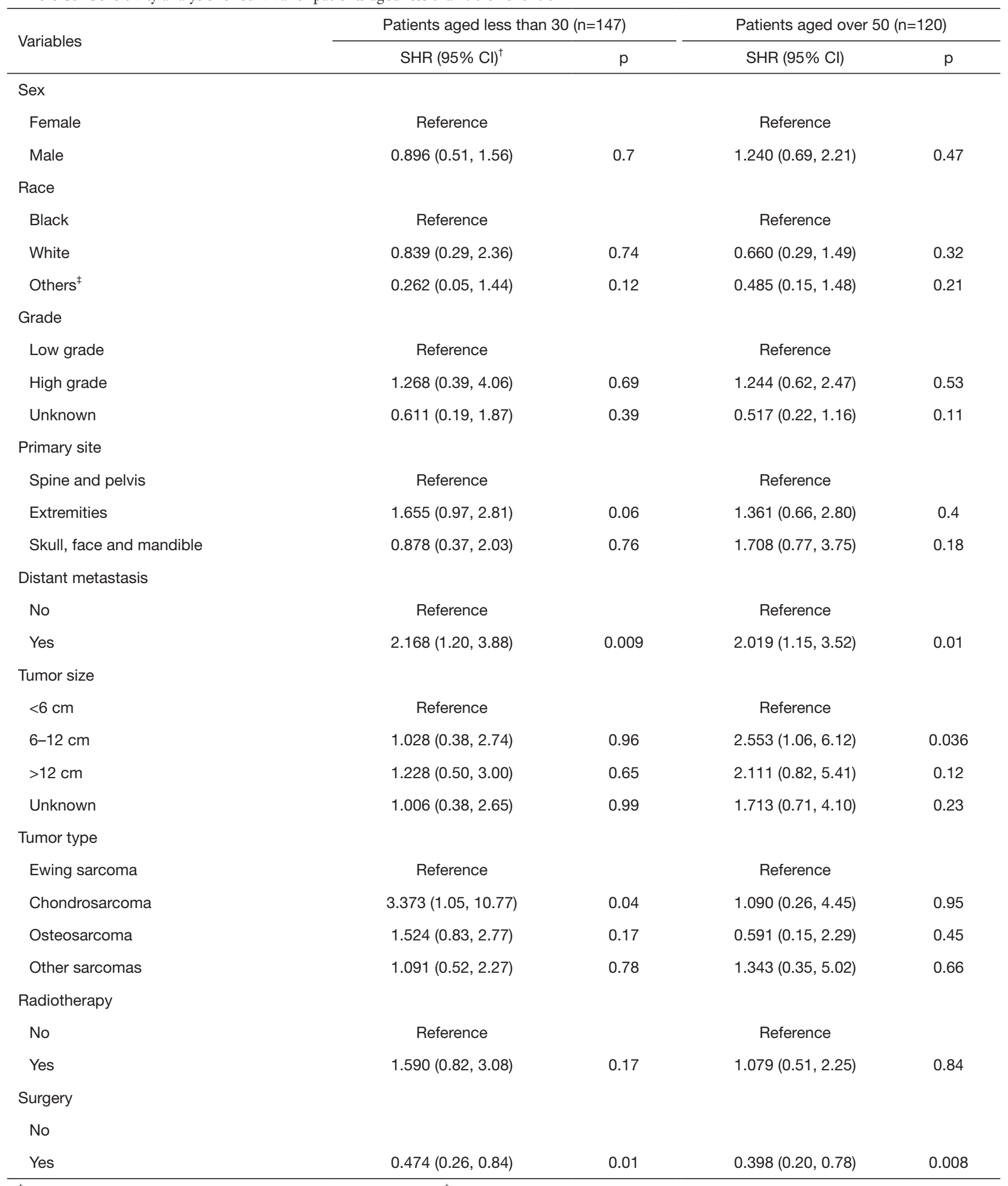

${ }^{\dagger}$ SHR: Sub-distribution hazard ratio; Cl: confidence interval; ${ }^{\ddagger}$ American Indian/Alaska Native, Asian/Pacific Islander 